\title{
A DEA MODEL FOR TWO-STAGE PARALLEL-SERIES PRODUCTION PROCESSES
}

\author{
Alireza Amirteimoori ${ }^{1}$ And Feng YAnG $^{2}$
}

\begin{abstract}
Data envelopment analysis (DEA) has been widely used to measure the performance of the operational units that convert multiple inputs into multiple outputs. In many real world scenarios, there are systems that have a two-stage network process with shared inputs used in both stages of productions. In this paper, the problem of evaluating the efficiency of a set of specialized and interdependent components that make up a large DMU is considered. In these processes the first stage consists of two parallel components which are connected serially with the process in the second stage. The paper develops a DEA approach for measuring efficiency of decision processes which can be divided into two stages. This application of parallel-series production process involves shared resources and the paper determines an optimal split of shared resources among two components.
\end{abstract}

Keywords. Data envelopment analysis, efficiency, production, two-stage.

Mathematics Subject Classification. 90B030.

\section{INTRODUCTION}

In the past few years, the data envelopment analysis (DEA) approach has become increasingly popular in the practice and research of efficiency analysis. Many DEA applications and research have led to new developments in concepts and

Received Mai 6, 2011. Accepted October 16, 2013.

1 Department of Applied Mathematics, Islamic Azad University, Rasht-Iran. aamirtemoori@gmail.com

2 School of Management, University of Science \& Technology of China, He Fei, An Hui Province, 230026, P.R. China. teimoori@guilan.ac.ir 
methodologies related to the DEA-efficiency analysis. Traditional DEA models consider DMUs with multiple inputs and multiple outputs (see [3]). However, as discussed in many DEA studies, DMUs can have a two-stage structure where the first stage uses inputs to produce outputs that then become the inputs to the second stage. The second stage thus consumes these first stage outputs to produce its outputs.

The issue of network DEA has been extensively studied. Recently, important steps toward the development of two-stage DEA have been taken by Färe and Grosskopf [10], Seiford and Zhu [16], Lothgern and Tambour [15], Färe and Grosskopf [11], Cook et al. [8], Hoopes et al. [13], Zhu [18], Chen and Zhu [5], Kao [14], Chen et al. [4,6], Tone and Tsutsi [17], Chen et al. [7], Cook et al. [9] and Amirteimoori [1]. Seiford and Zhu [16] introduced the two-stage processes and applied the standard DEA model to each stage. However, as noted in $[5,18]$, such an approach may conclude that two inefficient stages lead to an overall efficient DMU with the inputs of the first stage and outputs of second stage. Golany et al. [12] developed an efficiency measurement framework for systems composed of two subsystems arranged in series. Their approach expands the technology sets of each subsystem by allowing each to acquire resources from the other in exchange for delivery of the appropriate products, and to form composites from both subsystems.

Kao (2008) developed a parallel DEA model to measure the efficiency of the system which is composed of parallel production units. Chen et al. [4] proposed an additive efficiency decomposition approach wherein the overall efficiency is expressed as a weighted sum of the efficiencies of the individual stage. Chen et al. [6] examined relations and equivalent between the existing DEA approaches for measuring the performance of two-stage processes. Tone and Tsutsui (2009) proposed a network DEA model based on the weighted slack-based measure approach which accounts for the importance of each component. Chen et al. [7] developed a set of DEA models for measuring the performance of two-stage network processes with non splittable shared inputs. Additive efficiency decomposition for the two-stage network process was presented. Cook et al. [9] examined the more general problem of an open multistage process. In their paper, some outputs from a given stage may leave the system while others become inputs to the next stage. As well, new inputs can enter at any stage. They represented the overall efficiency of such a structure as an additive weighted average of the efficiencies of the individual components or stages that make up that structure. Chen et al. [7] developed a set of DEA models for measuring the performance of two-stage network processes with non splittable shared inputs.

This paper develops a parallel-series DEA model for measuring efficiency of decision processes which can be divided into two stages. In these processes, the first stage consists of two parallel production lines connected serially with the process in an assembly line. The two production lines use their own inputs and a shared input to generate two types of outputs which become inputs to the assembly line. The assembly line is then fed by a mixture of these two outputs and its own inputs 


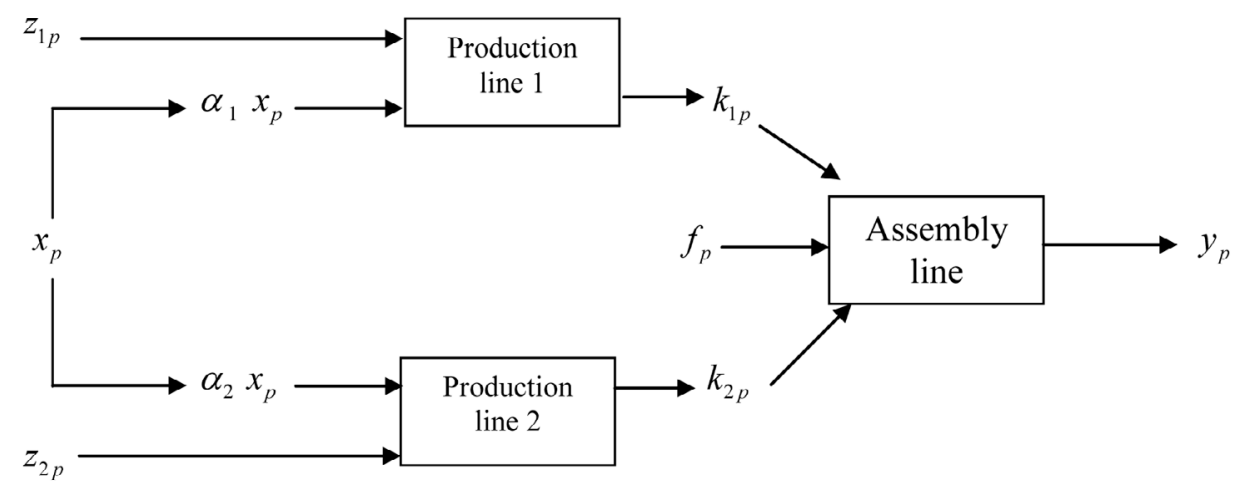

Figure 1. The production system.

to produce the final products. The structure in the current paper is different from those two-stage processes in the papers mentioned above, and the previous models are not suitable here. Additive efficiency decomposition for production lines and the assembly line is presented. The model proposed in this paper, determines a best resource split to optimize the additive efficiency of the whole system. We show herein that the presence of shared resources leads to a linear model rather than a nonlinear model.

The structure of the paper is organized as follows. The next section presents the proposed two-stage model. Section 3 applies the new approach to the 17 prefabricated cabin plants. Conclusions follow in Section 4.

\section{A TWO STAGE MODEL}

Consider a two-stage production process shown in Figure 1. Suppose we have n DMUs and each $D M U_{p}: p=1, \ldots, n$ consists of two parallel production lines and an assembly line. The first and second production lines use their own inputs $z_{p}^{(1)}=\left(z_{1 p}^{(1)}, z_{2 p}^{(1)}, \ldots, z_{D p}^{(1)}\right)^{T}$ and $z_{p}^{(2)}=\left(z_{1 p}^{(2)}, \ldots, z_{2 p}^{(2)}, \ldots, z_{H p}^{(2)}\right)^{T}$, respectively. We also assume that $D M U_{p}$ has $m$ inputs $x_{p}=\left(x_{1 p}, x_{2 p}, x_{m p}\right)^{T}$ that should be shared among the two production lines. The observed shared input to production lines 1 and 2 are respectively, $x_{p}^{(1)}=\left(x_{1 p}^{(1)}, x_{2 p}^{(1)}, \ldots, x_{m p}^{(1)}\right)^{T}$ and $x_{p}^{(2)}=\left(x_{1 p}^{(2)}, x_{2 p}^{(2)}, \ldots, x_{m p}^{(2)}\right)^{T}$ and obviously we have $x_{p}=x_{p}^{(1)}+x_{p}^{(2)}$. In optimality, some portion $0 \leq \alpha_{i}^{(1)}<1$ of the shared inputs $x_{i p}$ is allocated to the first line, and the remainder $0 \leq \alpha_{i}^{(2)}<1$ is allocated to the second line with $\alpha_{i}^{(1)}+\alpha_{i}^{(2)}=1$. So, the first component uses inputs $\alpha_{i}^{(1)} x_{i p}: i=1,2, \ldots, m$ and $z_{d p}^{(1)}: d=1,2, \ldots, D$ to produce $k_{l p}^{(1)}: l=1,2, \ldots, L$ and the second line uses $\alpha_{i}^{(2)} x_{i p}: i=1,2, \ldots, m$ and $z_{h p}^{(2)}: h=1,2, \ldots, H$ to produce $k_{b p}^{(2)}: b=1,2, \ldots, B$. The assembly line is fed by a mixture of inputs $k_{p}^{(1)}, k_{p}^{(2)}$ and an external input $f_{p}=\left(f_{1 p}, f_{2 p}, \ldots, f_{Q p}\right)^{T}$ 
and the final product is $y_{p}=\left(y_{1 p}, y_{2 p}, \ldots, y_{s p}\right)^{T}$. Let $\alpha^{(d)}=\left(\alpha_{1}^{(d)}, \alpha_{2}^{(d)}, \ldots, \alpha_{m}^{(d)}\right)^{T}$ : $i=1,2$.

Our object is to determine the relative efficiencies of the two production lines and assembly line along with an overall efficiency of the whole system. An Algebraic representation of the production possibility set of technology under consideration for the production line $d: d=1,2$ in constant returns to scale environment is defined as:

$$
\begin{gathered}
T_{d}=\left\{\left(\alpha^{(d)} \operatorname{diag}(x), z^{(d)}, k^{(d)}\right):\left(\alpha^{(d)} \operatorname{diag}(x)\right)^{T} \geq \sum_{j=1}^{n} \lambda_{j} x_{j}^{(d)}, z^{(d)} \geq \sum_{j=1}^{n} \lambda_{j} z_{j}^{(d)},\right. \\
\left.k^{(d)} \leq \sum_{j=1}^{n} \lambda_{j} k_{j}^{(d)}, 0 \leq \alpha^{(d)} \leq e^{T}, \lambda_{j} \geq 0, j=1,2, \ldots, n\right\}
\end{gathered}
$$

in which $\operatorname{diag}(x)$ is a diagonal matrix with diagonal elements $x_{1}, x_{2}, \ldots, x_{m}$ and $e^{T}=(1,1, \ldots, 1)$.

The $\operatorname{symbol} \operatorname{diag}(x)$ is used to define the products $\alpha_{i}^{(d)} x_{i}$ in matrix form as follows:

$\alpha^{(d)} \operatorname{diag}(x)=\left(\alpha_{1}^{(d)}, \alpha_{2}^{(d)}, \ldots, \alpha_{m}^{(d)}\right)\left[\begin{array}{cccc}x_{1} & 0 & \ldots & 0 \\ 0 & x_{2} & \ldots & 0 \\ \vdots & \vdots & \vdots & \vdots \\ 0 & 0 & \ldots & x_{m}\end{array}\right]=\left(\alpha_{1}^{(d)} x_{1}, \alpha_{2}^{(d)} x_{2}, \ldots, \alpha_{m}^{(d)} x_{m}\right)$.

Also, the superscript $t$ in $\left(\alpha^{(d)} \operatorname{diag}(x)\right)^{t}$ is used to transpose of the matrix $\alpha^{(d)} \operatorname{diag}(x)$. Now, let $T_{A}$ be the production possibility set of technology under consideration for the assembly line. $T_{A}$ is defined as follows:

$$
\begin{array}{r}
T_{A}=\left\{\left(k^{(1)}, k^{(2)}, f, y\right): k^{(1)} \geq \sum_{j=1}^{n} \lambda_{j} k_{j}^{(1)}, k^{(2)} \geq \sum_{j=1}^{n} \lambda_{j} k_{j}^{(2)},\right. \\
\left.f \geq \sum_{j=1}^{n} \lambda_{j} f_{j}, y \leq \sum_{j=1}^{n} \lambda_{j} y_{j}, \lambda_{j} \geq 0, j=1,2, \ldots, n\right\} .
\end{array}
$$

In applying the model described herein, attention is paid to additive model. In the assessment of production lines 1 and 2, the output measures $k^{(1)}$ and $k^{(2)}$ should be increased. On the other hand, these measures are considered as inputs to the assembly line and they should be decreased. If we treat the system's operation as a black-box, ignoring the intermediate measures may yield to an efficient DMU with inefficient production lines and/or assembly line. In model we proposed, the intermediate measures $k^{(1)}$ and $k^{(2)}$ are considered to be free variables, and they will be increased or decreased to make the whole system as efficient. To provide for a realistic picture of DMU's performance, some restrictions are imposed on the variables $\alpha^{(1)}$ and $\alpha^{(2)}$. Ratio constraints of the form $l_{i}^{(\alpha)} \leq \frac{\alpha_{i}^{(1)}}{\alpha_{i}^{(2)}} \leq u_{i}^{(\alpha)}$ on the 
portion variables $\alpha_{i}^{(1)}$ and $\alpha_{i}^{(2)}$ are imposed. These constraints reflect the relative importance of the shared resources that are split between two production lines.

Consider the assessment of $D M U_{p}: p \in\{1,2, \ldots, n\}$ in additive form. Taking in to consideration the forms of $T_{1}, T_{2}$ and $T_{A}$ and considering $\alpha_{i}^{(1)}+\alpha_{i}^{(2)}=1$ : $i=1,2, \ldots, m$, this is obtained as the optimal value of the following model:

$$
\begin{aligned}
\operatorname{Max} E_{p}= & e^{T} s^{\left(x^{(1)}\right)}+e^{T} s^{\left(x^{(2)}\right)}+e^{T} s^{\left(k_{1}\right)}+e^{T} s^{\left(z_{1}\right)}+e^{T} s^{\left(z_{2}\right)}+e^{T} s^{\left(k_{2}\right)} \\
& +e^{T} s^{(f)}+e^{T} s^{(y)}
\end{aligned}
$$

s.t.

Line 1 :

$$
\begin{aligned}
& \sum_{j=1}^{n} \lambda_{j} x_{j}^{(1)}+s^{\left(x^{(1)}\right)}=\left(\alpha^{(1)} \operatorname{diag}\left(x_{p}^{(1)}+x_{p}^{(2)}\right)\right)^{t}, \\
& \sum_{j=1}^{n} \lambda_{j} z_{j}^{(1)}+s^{\left(z^{(1)}\right)}=z_{p}^{(1)}, \\
& \sum_{j=1}^{n} \lambda_{j} k_{j}^{(1)}+s^{\left(k^{(1)}\right)}=k_{p}^{(1)} .
\end{aligned}
$$

Line 2 :

$$
\begin{aligned}
& \sum_{j=1}^{n} \lambda_{j} x_{j}^{(2)}+s^{\left(x^{(2)}\right)}=\left(\alpha^{(2)} \operatorname{diag}\left(x_{p}^{(1)}+x_{p}^{(2)}\right)\right)^{t}, \\
& \sum_{j=1}^{n} \lambda_{j} z_{j}^{(2)}+s^{\left(z^{(2)}\right)}=z_{p}^{(2)} \\
& \sum_{j=1}^{n} \lambda_{j} k_{j}^{(2)}+s^{\left(k^{(2)}\right)}=k_{p}^{(2)} .
\end{aligned}
$$

Assembly Line:

General constraint :

$$
\begin{aligned}
& \sum_{j=1}^{n} \lambda_{j} k_{j}^{(1)}+s^{\left(k^{(1)}\right)}=k_{p}^{(1)}, \\
& \sum_{j=1}^{n} \lambda_{j} k_{j}^{(2)}+s^{\left(k^{(2)}\right)}=k_{p}^{(2)}, \\
& \sum_{j=1}^{n} \lambda_{j} f_{j}+s^{(f)}=f_{p}, \\
& \sum_{j=1}^{n} \lambda_{j} y_{j}-s^{(y)}=y_{p} .
\end{aligned}
$$

$$
\begin{aligned}
& l_{i}^{(\alpha)} \leq \frac{\alpha_{i}^{(1)}}{\alpha_{i}^{(2)}} \leq u_{i}^{(\alpha)}, \\
& \alpha^{(1)}+\alpha^{(2)}=e, \\
& \alpha^{(1)}, \alpha^{(2)}, \lambda_{j} \geq 0, \text { for all } j, \\
& s^{\left(x^{(1)}\right)}, s^{\left(z^{(1)}\right)}, s^{\left(x^{(2)}\right)}, s^{\left(z^{(2)}\right)}, s^{(f)}, s^{(y)} \geq 0 .
\end{aligned}
$$


Theorem 2.1. The LP model (1) is feasible.

Proof. Clearly,

$$
\begin{aligned}
\lambda_{p} & =1, \lambda_{j}=0, j=1,2, \ldots, n, j \neq p, \\
s^{\left(x^{(1)}\right)} & =s^{\left(z^{(1)}\right)}=s^{\left(k^{(1)}\right)}=s^{\left(x^{(2)}\right)}=s^{\left(z^{(2)}\right)}=s^{\left(k^{(2)}\right)}=s^{(f)}=s^{(y)}=0, \\
\alpha_{i}^{(1)} & =\frac{x_{i p}^{(1)}}{x_{i p}^{(1)}+x_{i p}^{(2)}}, \quad i=1,2, \ldots, m, \\
\alpha_{i}^{(2)} & =\frac{x_{i p}^{(2)}}{x_{i p}^{(1)}+x_{i p}^{(2)}}, \quad i=1,2, \ldots, m,
\end{aligned}
$$

is a feasible solution to this problem.

Definition 2.2. $D M U_{p}$ is said to be additive efficient if and only if $E_{p}=0$.

Definition 2.3. $D M U_{p}$ is said to be additive efficient in stage 1 if and only if $E_{p}^{(1)}=e^{T} s^{\left(x^{(1)}\right)}+e^{T} s^{\left(k_{1}\right)}+e^{T} s^{\left(z_{1}\right)}=0$.

Definition 2.4. $D M U_{p}$ is said to be additive efficient in stage 2 if and only if $E_{p}^{(2)}=e^{T} s^{\left(x^{(2)}\right)}+e^{T} s^{\left(z_{2}\right)}+e^{T} s^{\left(k_{2}\right)}=0$.

Definition 2.5. $D M U_{p}$ is said to be additive efficient in assembly line if and only if $E_{p}^{(A)}=e^{T} s^{\left(k_{1}\right)}+e^{T} s^{\left(k_{2}\right)}+e^{T} s^{(f)}+e^{T} s^{(y)}=0$.

For an inefficient production line $d: d=1,2$, we have $E_{p}^{(d)}>0$. In this case, we must have

$$
\begin{aligned}
& x_{p}^{(d)}=\sum_{j=1}^{n} \lambda_{j} x_{j}^{(d)}+s^{\left(x^{(d)}\right)}+\left[x_{p}^{(d)}-\alpha^{(d)} \operatorname{diag}\left(x_{p}^{(1)}+x_{p}^{(2)}\right)\right], \\
& z_{p}^{(1)}=\sum_{j=1}^{n} \lambda_{j} z_{j}^{(1)}+s^{\left(z^{(1)}\right)}, \\
& k_{p}^{(1)}=\sum_{j=1}^{n} \lambda_{j} k_{j}^{(1)}+s^{\left(k^{(1)}\right)},
\end{aligned}
$$


When $E_{p}^{(A)}>0$, the $p$ th assembly line is inefficient and we have

$$
\begin{aligned}
k_{p}^{(1)} & =\sum_{j=1}^{n} \lambda_{j} k_{j}^{(1)}+s^{\left(k^{(1)}\right)}, \\
k_{p}^{(2)} & =\sum_{j=1}^{n} \lambda_{j} k_{j}^{(2)}+s^{\left(k^{(2)}\right)}, \\
f_{p} & =\sum_{j=1}^{n} \lambda_{j} f_{j}+s^{(f)}, \\
y_{p} & =\sum_{j=1}^{n} \lambda_{j} y_{j}-s^{(y)} .
\end{aligned}
$$

The production and assembly lines can be improved and become efficient by deleting the input excess and augmenting the output shortfalls.

It is to be noted that the intermediate measures $k^{(1)}$ and $k^{(2)}$ may be increased or decreased to make the whole system as efficient. These operations are called CRS projection (constant returns to scale) and make the inefficient process as efficient.

Generalization of the model.

In model 1, we assumed there are only two production stations in the first stage. Consider now that there are $\mathrm{G}$ parallel production stations and the $g$ th station uses inputs $\alpha_{i}^{(g)} x_{i p}: i=1,2, \ldots, m$ and $z_{(h p)}^{(g)}: \quad h=1,2, \ldots, H_{g}$ to generate $k_{(l p)}^{(g)}: \quad l=1,2, \ldots, L_{g}$. The production possibility set of technology under consideration for production line $g: g=1,2, \ldots, G$ is as follows:

$$
\begin{gathered}
T_{g}=\left\{\left(\alpha^{(g)} \operatorname{diag}(x), z^{(g)}, k^{(g)}\right):\left(\alpha^{(g)} \operatorname{diag}(x)\right)^{T} \geq \sum_{j=1}^{n} \lambda_{j} x_{j}^{(g)}, z^{(g)} \geq \sum_{j=1}^{n} \lambda_{j} z_{j}^{(g)},\right. \\
\left.k^{(g)} \leq \sum_{j=1}^{n} \lambda_{j} k_{j}^{(g)}, 0 \leq \alpha^{(g)} \leq e^{T}, \lambda_{j} \geq 0, j=1,2, \ldots, n\right\} .
\end{gathered}
$$

Moreover, the production possibility set of technology under consideration for the assembly line is defined as follows:

$$
\begin{gathered}
T_{A}=\left\{\left(k^{(1)}, k^{(2)}, \ldots, k^{(G)}, f, y\right): k^{(g)} \geq \sum_{j=1}^{n} \lambda_{j} k_{j}^{(g)}, g=1,2, \ldots, G,\right. \\
\left.f \geq \sum_{j=1}^{n} \lambda_{j} f_{j}, y \leq \sum_{j=1}^{n} \lambda_{j} y_{j}, \lambda_{j} \geq 0, j=1,2, \ldots, n\right\} .
\end{gathered}
$$


Taking in to account the forms of $T_{g}: g=1,2, \ldots, G$ and $T_{A}$ and considering $\sum_{g=1}^{G} \alpha_{i}^{(g)}=1, i=1,2, \ldots, m$, this is obtained as the optimal value of the following model:

$\operatorname{Max} E_{p}=\sum_{g=1}^{G} e^{T} s^{\left(x^{(g)}\right)}+\sum_{g=1}^{G} e^{T} s^{\left(k_{g}\right)}+\sum_{g=1}^{G} e^{T} s^{\left(z_{g}\right)}+e^{T} s^{(f)}+e^{T} s^{(y)}$ s.t.

Production line $g: g=1,2, \ldots, G$ :

$$
\begin{aligned}
& \sum_{j=1}^{n} \lambda_{j} x_{j}^{(g)}+s^{\left(x^{(g)}\right)}=\left(\alpha^{(g)} \operatorname{diag}\left(\sum_{g=1}^{G} x_{p}^{(g)}\right)^{t},\right. \\
& \sum_{j=1}^{n} \lambda_{j} z_{j}^{(g)}+s^{\left(z^{(g)}\right)}=z_{p}^{(g)}, \\
& \sum_{j=1}^{n} \lambda_{j} k_{j}^{(g)}+s^{\left(k^{(g)}\right)}=k_{p}^{(g)} .
\end{aligned}
$$

Assembly line:

$$
\begin{aligned}
& \sum_{j=1}^{n} \lambda_{j} k_{j}^{(g)}+s^{\left(k^{(g)}\right)}=k_{p}^{(g)}, g=1,2, \ldots, G, \\
& \sum_{j=1}^{n} \lambda_{j} f_{j}+s^{(f)}=f_{p}, \\
& \sum_{j=1}^{n} \lambda_{j} y_{j}-s^{(y)}=y_{p} .
\end{aligned}
$$

General constraint:

$$
\begin{aligned}
& l^{(\alpha)} \leq \frac{\alpha^{(i)}}{\alpha^{(j)}} \leq u^{(\alpha)}, \text { for all } i \text { and } j, \\
& \sum_{g=1}^{G} \alpha^{(g)}=1, \\
& \alpha^{(g)}, \lambda_{j} \geq 0, \text { for all } g \text { and } j, \\
& s^{\left(x^{(g)}\right), s^{\left(z^{(g)}\right)}, s^{(f)}, s^{(y)} \geq 0 .}
\end{aligned}
$$


TABLE 1. The input-output measures used in this application.

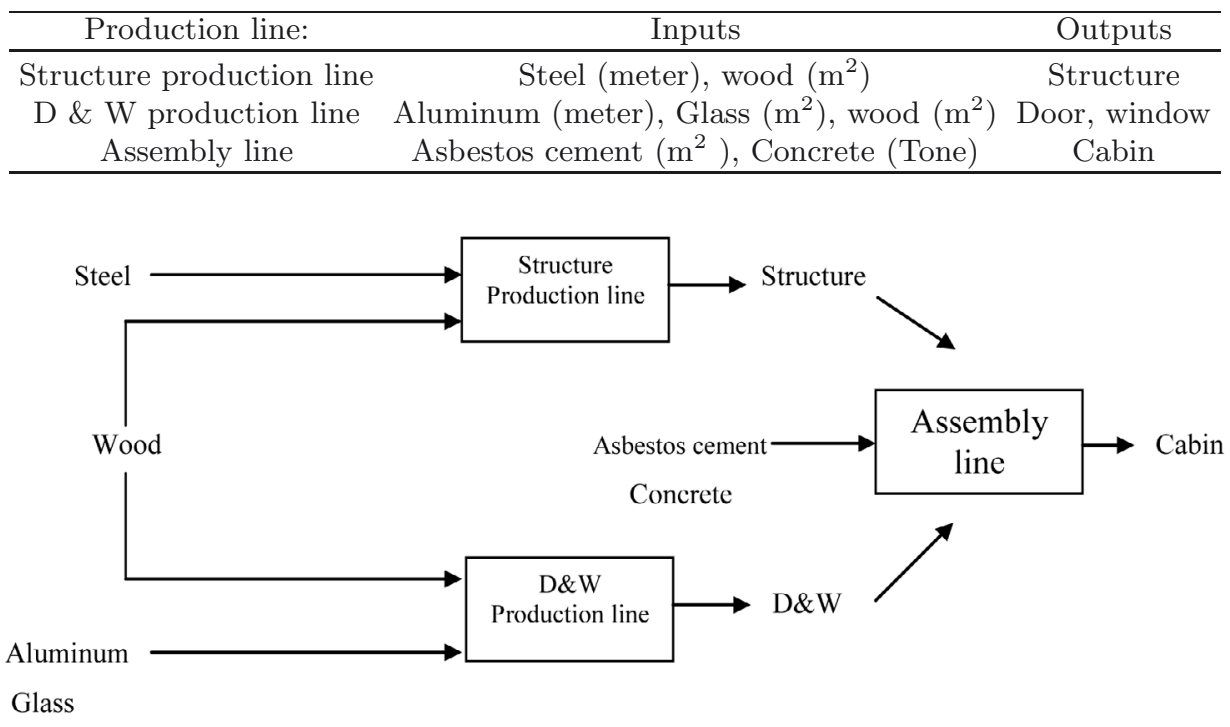

FIGURE 2. The production process of prefabricated cabin.

\section{Application}

In this section, we apply the two-stage production process discussed in this paper with the analysis of manufacturing company's activities. A limited company in Golestan, Iran, has 17 plants that produce prefabricated cabins. Each manufactory consists of two production lines arranged in series: structure production line and doors and windows $(D \& W)$ production line. The structure production line uses steels $\left(z_{1}^{(1)}\right)$ and some portion of woods $\left(x_{1}\right)$ to produce structures $\left(k^{(1)}\right)$. Parallel to this line, the $D \& W$ production line uses glasses $\left(z_{2}^{(2)}\right)$, some portion of woods $\left(x_{2}\right)$ and aluminums $\left(z_{1}^{(2)}\right)$ to produce doors and windows $\left(k^{(2)}\right)$. The produced structures, doors and windows will be assembled in the assembly line to produce the final products that are prefabricated cabins $(y)$. The assembly line uses two external inputs: corrugated plate (Asbestos cement) $\left(f_{1}\right)$ and concrete $\left(f_{2}\right)$.

The input-output measures that are used in this application are summarized in Table 1.

The production process is depicted in Figure 2. The data for a six-month period is displayed in Table 2 . The results from model (1) are reported in Table 3 where the columns are the inefficiency slacks obtained from model 1. As the table indicates, eight plants are efficient in overall sense. The projection points are listed in Table 4 (note that incomplete projects are acceptable by the board of management and hence, the input/output measures are not restricted to take integer values). 
TABle 2. Plants data.

\begin{tabular}{ccccccccccc}
\hline$D M U_{j}$ & $x^{(1)}$ & $x^{(2)}$ & $z_{1}^{(1)}$ & $z_{1}^{(2)}$ & $z_{2}^{(2)}$ & $k^{(1)}$ & $k^{(2)}$ & $y_{1}$ & $f_{1}$ & $f_{2}$ \\
\hline 1 & 5 & 5.5 & 12700 & 9 & 8 & 168 & 328 & 160 & 310 & 1600 \\
2 & 4.6 & 4.4 & 11500 & 9 & 4 & 120 & 226 & 108 & 290 & 1100 \\
3 & 3.5 & 5 & 11300 & 11 & 7 & 84 & 220 & 84 & 160 & 800 \\
4 & 2 & 3.2 & 8000 & 12 & 6 & 65 & 156 & 69 & 165 & 750 \\
5 & 6.3 & 3.7 & 13000 & 12 & 11 & 144 & 268 & 132 & 260 & 1400 \\
6 & 4.7 & 5.8 & 13500 & 22 & 23 & 158 & 280 & 149 & 290 & 1500 \\
7 & 4.3 & 5.2 & 12000 & 29 & 31 & 144 & 268 & 130 & 230 & 1350 \\
8 & 6.8 & 4.2 & 13450 & 13 & 9 & 168 & 326 & 158 & 300 & 1600 \\
9 & 5 & 3.5 & 11010 & 28 & 11 & 120 & 240 & 112 & 260 & 1100 \\
10 & 4.1 & 3.4 & 10500 & 19 & 12 & 89 & 178 & 84 & 160 & 900 \\
11 & 4.8 & 5.2 & 12350 & 10 & 9 & 144 & 284 & 132 & 235 & 1300 \\
12 & 4.4 & 5.6 & 13000 & 29 & 17 & 144 & 262 & 129 & 225 & 1350 \\
13 & 3.8 & 4.2 & 11505 & 9 & 11 & 108 & 200 & 99 & 215 & 1000 \\
14 & 5 & 3.5 & 9550 & 22 & 21 & 96 & 178 & 82 & 165 & 850 \\
15 & 5.2 & 6.3 & 13800 & 24 & 11 & 168 & 330 & 157 & 315 & 1600 \\
16 & 5.4 & 5.1 & 13500 & 22 & 21 & 141 & 312 & 144 & 300 & 1500 \\
17 & 6.8 & 5.7 & 13505 & 24 & 11 & 153 & 318 & 150 & 295 & 1550 \\
\hline
\end{tabular}

TABLE 3. Results from model 1.

\begin{tabular}{ccccccc}
\hline$D M U_{j}$ & $E_{p}$ & $E_{p}^{(1)}$ & $E_{p}^{(2)}$ & $E_{p}^{(A)}$ & $\alpha_{1}$ & $\alpha_{2}$ \\
\hline $\mathrm{DMU}$ & & & & & & \\
1 & 0 & 0 & 0 & 0 & 0.4762 & 0.5238 \\
2 & 0 & 0 & 0 & 0 & 0.5111 & 0.4889 \\
3 & 0 & 0 & 0 & 0 & 0.4118 & 0.5882 \\
4 & 2632.8781 & 2516.3469 & 25.2187 & 98.4125 & 0.5439 & 0.4561 \\
5 & 2619.8625 & 2529.2375 & 6.375 & 87.05 & 0.5462 & 0.4538 \\
6 & 0 & 0 & 0 & 0 & 0.4476 & 0.5524 \\
7 & 0 & 0 & 0 & 0 & 0.4526 & 0.5474 \\
8 & 387.9808 & 361.9602 & 1.3839 & 23.4801 & 0.4884 & 0.5116 \\
9 & 0 & 0 & 0 & 0 & 0.5882 & 0.4118 \\
10 & 3698.46 & 3622.8657 & 21.88 & 59.1829 & 0.6027 & 0.3973 \\
11 & 0 & 0 & 0 & 0 & 0.48 & 0.52 \\
12 & 0 & 0 & 0 & 0 & 0.44 & 0.56 \\
13 & 3692.1469 & 3652.4281 & 6.5312 & 34.2875 & 0.5746 & 0.4254 \\
14 & 3134.5812 & 3054.2687 & 44.1875 & 55.925 & 0.6684 & 0.3316 \\
15 & 1409.7531 & 1342.4719 & 26.4687 & 52.1125 & 0.5307 & 0.4693 \\
16 & 2186.35 & 2060.85 & 44.5 & 87.6 & 0.5286 & 0.4714 \\
17 & 1680.8438 & 1596.9063 & 29.5625 & 60.375 & 0.5875 & 0.4125 \\
\hline
\end{tabular}


TABLE 4. Projection points.

\begin{tabular}{ccccccccccc}
\hline$D M U_{j}$ & $x^{(1)}$ & $x^{(2)}$ & $z_{1}^{(1)}$ & $z_{1}^{(2)}$ & $z_{2}^{(2)}$ & $k^{(1)}$ & $k^{(2)}$ & $y_{1}$ & $f_{1}$ & $f_{2}$ \\
\hline 1 & 5 & 5.5 & 12700 & 9 & 8 & 168 & 328 & 160 & 310 & 1600 \\
2 & 4.6 & 4.4 & 11500 & 9 & 4 & 120 & 226 & 108 & 290 & 1100 \\
3 & 3.5 & 5 & 11300 & 11 & 7 & 84 & 220 & 84 & 160 & 800 \\
4 & 2.16 & 2.37 & 5476.88 & 3.88 & 3.45 & 72.45 & 141.45 & 69 & 133.69 & 690 \\
5 & 4.13 & 4.54 & 10477.5 & 7.43 & 6.6 & 138.6 & 270.6 & 132 & 255.75 & 1320 \\
6 & 4.7 & 5.8 & 13500 & 22 & 23 & 158 & 280 & 149 & 290 & 1500 \\
7 & 4.3 & 5.2 & 12000 & 29 & 31 & 144 & 268 & 130 & 230 & 1350 \\
8 & 5.13 & 5.63 & 13088.76 & 9.99 & 9 & 167.53 & 327.63 & 158 & 300 & 1575.36 \\
9 & 5 & 3.5 & 11010 & 28 & 11 & 120 & 240 & 112 & 260 & 1100 \\
10 & 2.65 & 2.98 & 6878.86 & 7.45 & 7.28 & 89.14 & 172.39 & 84 & 160 & 846.29 \\
11 & 4.8 & 5.2 & 12350 & 10 & 9 & 144 & 284 & 132 & 235 & 1300 \\
12 & 4.4 & 5.6 & 13000 & 29 & 17 & 144 & 262 & 129 & 225 & 1350 \\
13 & 3.09 & 3.4 & 7858.13 & 5.57 & 4.95 & 103.95 & 202.95 & 99 & 191.81 & 990 \\
14 & 2.56 & 2.82 & 6508.75 & 4.61 & 4.1 & 86.1 & 168.1 & 82 & 158.88 & 820 \\
15 & 4.91 & 5.4 & 12461.88 & 8.83 & 7.85 & 164.85 & 321.85 & 157 & 304.19 & 1570 \\
16 & 4.5 & 4.95 & 11430 & 8.1 & 7.2 & 151.2 & 295.2 & 144 & 279 & 1440 \\
17 & 4.69 & 5.16 & 11906.25 & 8.44 & 7.5 & 157.5 & 307.5 & 150 & 290.63 & 1500 \\
\hline
\end{tabular}

The interpretation of our model can be illustrated by considering a specific plant, say plant 10 . The production lines 1 and 2 and the assembly line are inefficient in this plant. The shared resource to this plant should be reduced from its current level 7.5 (4.1 for production line 1 and 3.4 for the second line) to 5.63 (2.65 for production line 1 and 2.98 for the second line). The projection point to this plant is

$$
\begin{aligned}
& \left(x^{(1)}, x^{(2)}, z_{1}^{(1)}, z_{1}^{(2)}, z_{2}^{(2)}, k^{(1)}, k^{(2)}, y_{1}, f_{1}, f_{2}\right)= \\
& (2.65,2.98,6878.86,7.45,7.28,89.14,172.39,84,160,846.29) .
\end{aligned}
$$

Considering the optimal values to $\alpha_{1}$ and $\alpha_{2}$, we conclude that the first intermediate measure $k^{(1)}$ should be increased from 89 to 89.14 , whereas, the second one, $k^{(2)}$, should be decreased from 178 to 172.39 . These reductions make the whole chain as efficient.

\section{Conclusion}

This paper discusses the efficiency measurement of two-stage production processes with three processes where two parallel processes in the first stage are connected serially with the process in the second stage. For this type of production system, an additive efficiency measure has been defined. A method for determining the DEA frontier points for inefficient components in these parallel-series production systems has been faced. This application of parallel-series production process 
involves shared resources and the model proposed in this paper, determines an optimal split of shared resources. The case of prefabricated cabin plants is given using this newly developed approach.

The DEA model discussed in this paper is under CRS, in other words, an assumption of constant returns to scale is considered. The approach is also applicable to variable returns to scale under the BCC model of Banker at al. [2] by including the constraint $\sum_{j=1}^{n} \lambda_{j}=1$ in the LP model 1.

Acknowledgements. The authors are grateful to the editor professor Ridha Mahjoub for ensuring a timely review process. Any errors and omissions are our own.

\section{REFERENCES}

[1] A. Amirteimoori, A DEA two-stage decision process with shared resources. Central Eur. J. Oper. Res. 21 (2013) 141-151.

[2] R.D. Banker, A. Charnes and W.W. Cooper, Some Methods for Estimating Technical and Scale Inefficiencies in Data Envelopment Analysis. Management Science 30 (1984) 10781092.

[3] A. Charnes, W.W. Cooper and E. Rhodes, Measuring the Efficiency of Decision Making Units. Eur. J. Oper. Res. 2 (1978) 429-444.

[4] Y. Chen, W.D. Cook, N. Li and J. Zhu, Additive Efficiency Decomposition in Two-Stage DEA. Eur. J. Oper. Res. 196 (2009a) 1170-1176.

[5] Y. Chen and J. Zhu, Measuring Information Technology's Indirect Impact on Firm Performance. Inf. Technol. Manag. J. 5 (2004) 9-22.

[6] Y. Chen, L. Liang and J. Zhu, Equivalence in two-stage DEA approaches. Eur. J. Oper. Res. 193 (2009b) 600-604.

[7] Y. Chen, J. Du, H.D. Sherman and J. Zhu, DEA model with shared resources and efficiency decomposition. Eur. J. Oper. Res. 207 (2010) 339-349.

[8] W.D. Cook, M. Hababou and H. Tuenter, Multi-component efficiency measurement and shared inputs in DEA: an application to sales and service performance in bank branches. $J$. Prod. Anal. 14 (2000) 209-224.

[9] W.D. Cook, J. Zhu, G. Bi and F. Yang, Network DEA: Additive efficiency decomposition. Eur. J. Oper. Res. 207 (2010) 1122-1129.

[10] R. Färe and S. Grosskopf, Productivity and intermediate products: A frontier approach, Econ. Lett. 50 (1996) 65-70.

[11] R. Färe and S. Grosskopf, Network DEA. Socio-Economic Planning Sciences 34 (2000) 35-49.

[12] B. Golany, S.T. Hackman and U. Passy, An Efficiency measurement Framework for Multistage Production System. Ann. Oper. Res. 145 (2006) 51-68.

[13] B. Hoopes, K.P. Triantis and N. Partangel, The Relationship Between Process and Manufacturing Plant Performance: A Goal Programming Approach. Int. J. Oper. Quantit. Manag. 6 (2000) 287-310.

[14] C. Kao, Efficiency Measurement for Parallel Production systems. Eur. J. Oper. Res. 196 (2009) 1107-1112.

[15] M. Lothgren and M. Tambour, Productivity and customer satisfaction in Swedish pharmacies: A DEA network model. Eur. J. Oper. Res. 115 (1999) 449-458.

[16] L.M. Seiford and J. Zhu, Profitability and Marketability of the Top 55 US Commercial Banks. Management Science 45 (1999) 1270-1288.

[17] K. Tone and M. Tsutsui, Network DEA: A slack-based measure approach. Eur. J. Oper. Res. 197 (2009) 243-252.

[18] J. Zhu, Quantitative Models for Performance Evaluation and Benchmarking: Data Envelopment Analysis with Spreadsheets. Kluwer Academic Publishers, Boston (2003). 